\title{
Surgical Treatment for Mesial Temporal Lobe Epilepsy Accompanied with Neuro-Behçet's Disease: A Case Report
}

\author{
Hirofumi IWAMOTO, ${ }^{1}$ Ryosuke HANAYA, ${ }^{1,2}$ Surya Pratama BRILliAnTIKA, ${ }^{1}$ \\ Masanori SATO, ${ }^{1,2}$ Hiroshi Hosoyama, ${ }^{1,2}$ Toshiaki OTSUBO, ${ }^{3}$ \\ Fujio UMEHARA, ${ }^{4}$ and Koji YOSHIMOTO ${ }^{1}$ \\ ${ }^{1}$ Department of Neurosurgery, Graduate School of Medicine, Kagoshima University, \\ Kagoshima, Kagoshima, Japan \\ ${ }^{2}$ Epilepsy Center, Kagoshima University Hospital, Kagoshima, Kagoshima, Japan \\ ${ }^{3}$ Fujimoto General Hospital, Miyakonojo, Miyazaki, Japan \\ ${ }^{4}$ Department of Neurology, Nanpuh Hospital, Kagoshima, Kagoshima, Japan
}

\begin{abstract}
Behçet's disease (BD) is a rare chronic inflammatory disease associated with systemic vasculitis. Involvement of the nervous system in BD is called neuro-BD (NBD). Epilepsy related to NBD is uncommon but responds well to anti-epileptic drugs. We present a case of NBD with drug-resistant mesial temporal lobe epilepsy (MTLE) due to hippocampal sclerosis (HS). The patient presented with headache, dizziness, disorientation, and generalized seizures. Magnetic resonance imaging (MRI) identified pontine lesions. Chronic inflammation was suspected, and steroid pulse therapy improved his symptoms. He relapsed 1 year after onset and was diagnosed with NBD. MRI revealed bilateral mesial temporal lesions, with the right being edematous and the left atrophic. NBD was controlled by steroid and immunosuppressive medication. Three years after the onset of NBD, the patient suffered MTLE, and MRI suggested left hippocampal atrophy. His seizures became drug-resistant and surgical therapy was considered 12 years after NBD onset. Pre-surgical MRI clearly showed left HS. After evaluations, the patient had left anterior temporal lobectomy (ATL) 13 years after NBD onset under stable NBD. The patient was seizure-free for $>2$ years after surgery. Surgery will be an effective treatment for drug-resistant MTLE with HS even in patients with NBD, of course the effects of surgical intervention should be considered.
\end{abstract}

Keywords: acute and chronic progressive types, anterior temporal lobectomy, hippocampal sclerosis, interleukin-6, parenchymal syndromes

\section{Introduction}

Behçet's disease (BD) is an inflammatory multisystemic disease of unknown etiology, with unpredictable exacerbations and remissions. ${ }^{1)}$ The prevalence of the disease is $14-20 / 100000$ in countries along the Silk Road, and 0.12-0.64/100000 in Western countries. BD develops between the ages of 20 and 40 years, and has variable clinical symptoms, such

Received June 29, 2020; Accepted November 6, 2020

Copyright $\odot 2021$ The Japan Neurosurgical Society This work is licensed under a Creative Commons AttributionNonCommercial-NoDerivatives International License. as recurrent oral and genital ulcers, uveitis, skin manifestation, arthritis, neurological manifestations, and hypercoagulable state. ${ }^{2)} \mathrm{BD}$ with neurological manifestations is referred to as Neuro-BD (NBD), and its frequency in patients with $\mathrm{BD}$ greatly varies from $1.3 \%$ to $59 \%{ }^{3)}$

Neurological syndromes recognized as NBD are classified into parenchymal syndromes and nonparenchymal syndromes. Almost $75 \%$ of NBD cases are parenchymal syndromes, which include brainstem lesion, multifocal (diffuse) lesion, myelopathy, and cerebral and optic neuropathy. Non-parenchymal syndromes include cerebral venous thrombosis, intracranial hypertension, and acute meningeal syndrome..$^{4)}$ Parenchymal NBD is further subdivided 
into acute and chronic progressive (CP) types..5) Acute NBD is characterized by acute meningoencephalitis with or without focal lesions. Acute NBD mostly responds to corticosteroid therapy with/ without cytotoxic agents, and is usually self-limiting, although recurrence of attacks may occur. CP-NBD is characterized by intractable, slowly progressive neurobehavioral changes, ataxia, and dysarthria, leading to severe disability and deterioration. CP-NBD is resistant to corticosteroids, cyclophosphamide, or azathioprine. Characteristic findings of each type have been extensively described ${ }^{3)}$ Fever commonly occurs in acute NBD while sphincter disturbances, ataxia (acute NBD, 16\%; CP-NBD, 57\%), dementia, and confusion are more common in CP-NBD. In the cerebrospinal fluid (CSF), cell count is higher in acute NBD (acute NBD, 94/ $\mathrm{mm}^{3}$; CP-NBD, 11/ $\mathrm{mm}^{3}$ ). MRI findings of brain stem atrophy and abnormal findings in cerebellum are more common in CP-NBD (acute NBD, 7\%; CP-NBD, 54\%).

Hippocampal sclerosis (HS) is typically seen in drug-resistant mesial temporal lobe epilepsy (MTLE), and surgery is more effective than drug treatment. ${ }^{6}$ However, the impact of surgical intervention for MTLE on NBD recurrence and exacerbation in patients with NBD is an important consideration. The impact of cranial surgery on patients with a history of NBD is yet to be defined. Further considerations include whether the intracranial lesion associated with relapse-remission has future epileptic potential, ${ }^{7)}$ and whether surgery can be of benefit in patients with CP type.

We report a patient with a rare case of NBD who received anterior temporal lobectomy (ATL) for drug-resistant MTLE.

\section{Case Report}

A 22-year-old man was referred to the nearby hospital due to headache, dizziness, and disorientation. At the hospital, he had generalized seizures. His past medical history was significant for recurrent oral ulcer and nodular erythema since childhood. He also had a family history of epilepsy. The patient grew up in an orphanage, which made it difficult to collect further detailed information about his family and his childhood, including the presence of perinatal abnormality and febrile convulsion. The medical record after his initial assessment reported that magnetic resonance imaging (MRI) revealed cerebellar hyperintensity lesion in T2-weighted image (T2WI). CSF had elevation of cells (35 cells/ $\mu \mathrm{L}$; reference, $0-5)$ and total protein $(69 \mathrm{mg} / \mathrm{dL}$; reference, 8-43), normal glucose level (60 mg/dL; reference, 50-75), and was negative for herpes simplex virus-deoxyribonucleic acid (DNA), tuberculosis-DNA, myelin basic protein, oligoclonal band, and cell culture. A diagnosis of NBD was considered owing to the past medical history. He received steroid pulse therapy, after which his symptoms improved.

Discontinuation of oral prednisone (30 mg/day) a year after discharge resulted in recurrence of headache and diplopia, and he was referred and admitted to our hospital. Examination revealed erythema on the left side of the neck and back scalp skin, and small papules in the genital region. MRI demonstrated high intensities in T2WI in the brain stem, cerebellum, right mesial temporal lobe, and bilateral basal ganglia (Fig. 1). This MRI also revealed atrophy of the left hippocampus. The erythema papules and elevated interleukin (IL)-6 (106 pg/mL; reference <4.0) in CSF were suggestive of BD, but a positive result for serum human leukocyte antigen (HLA)-B54 was characteristic of Sweet syndrome, which is a rare inflammatory skin condition characterized by sudden onset of fever and painful rash. Skin biopsy showed infiltration of inflammatory cells accompanied by vasculitis, which is typical of NBD. His condition was diagnosed as NBD from these results despite his symptoms not fulfilling the diagnostic criteria. Steroid therapy improved neurological symptoms and inflammatory markers in blood and CSF. MRI lesions in brain stem, cerebellum, and basal ganglia also resolved. He continued oral prednisolone (30 mg/day) and oral azathioprine $(100 \mathrm{mg} /$ day) as an out-patient.

Three years after first admission to our hospital, the patient presented with features suggestive of focal aware seizure (FAS) (the feeling of being driven to a corner), focal impaired awareness seizure (FIAS) (loss of consciousness and automatism), and focal to bilateral tonic clonic seizure (FBTCS) following FIAS. Electroencephalography (EEG) showed interictal discharge in the left temporal lobe, and MRI showed left hippocampal atrophy (Fig. 2a), which was similar to the previous imaging result (Fig. 1). A diagnosis of left MTLE was made, rather than an exacerbation of NBD, and sodium valproate (400 $\mathrm{mg} /$ day) was initiated. IL-6 in CSF was $20.9 \mathrm{pg} / \mathrm{mL}$, which indicated that the NBD could be chronic.

After years of follow-up, three antiepilepticssodium valproate (1000 mg/day), levetiracetam (2000 $\mathrm{mg} /$ day), and gabapentin (2400 $\mathrm{mg} /$ day)—failed to inhibit the seizures, and the patient continued experiencing FAS, including Deja vu twice weekly, FIAS three times a month, and FBTCS yearly. The severity of NBD did not correlate with frequency of seizures. He had to temporarily retire from work because of the frequent seizures and was referred to the epilepsy center to be evaluated for epilepsy 

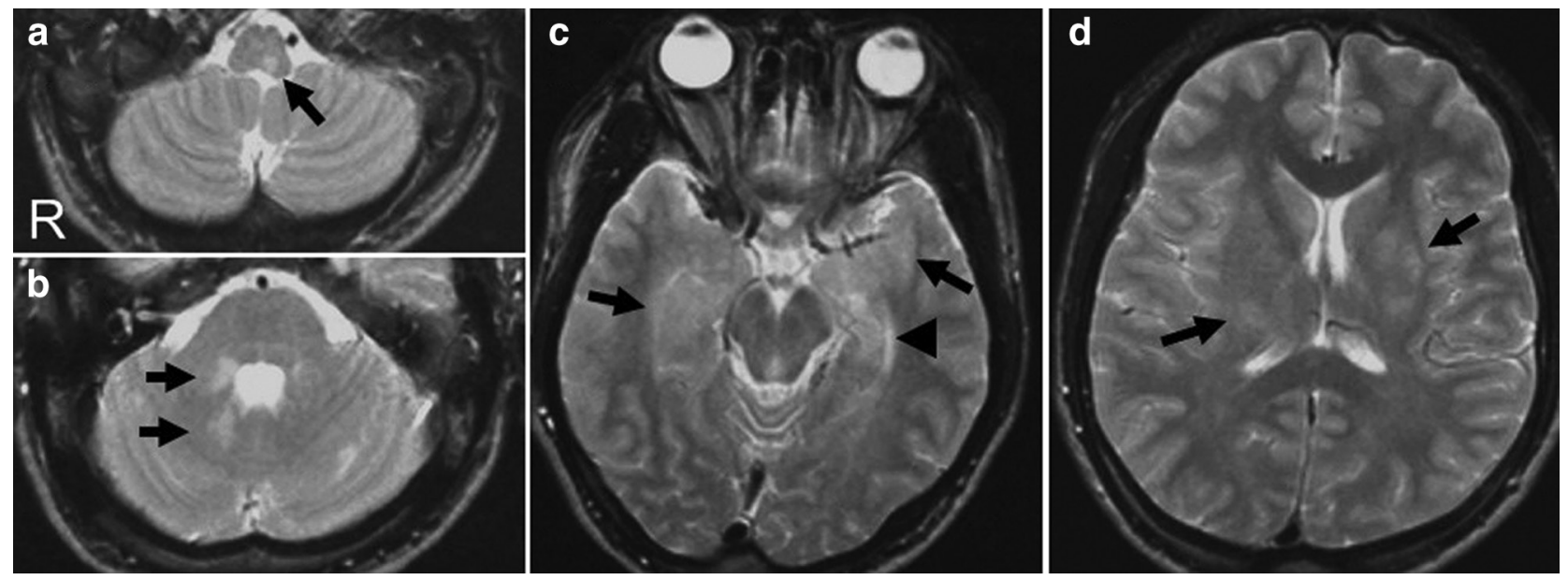

Fig. 1 MRI on second admission. Axial T2WI demonstrated high intensity lesions (arrow) in left brain stem (a), right cerebellum (b), left temporal lobe (c), and bilateral basal ganglia (d). Left hippocampus was atrophic (arrowhead) (c). MRI: magnetic resonance imaging, T2WI: T2-weighted image.
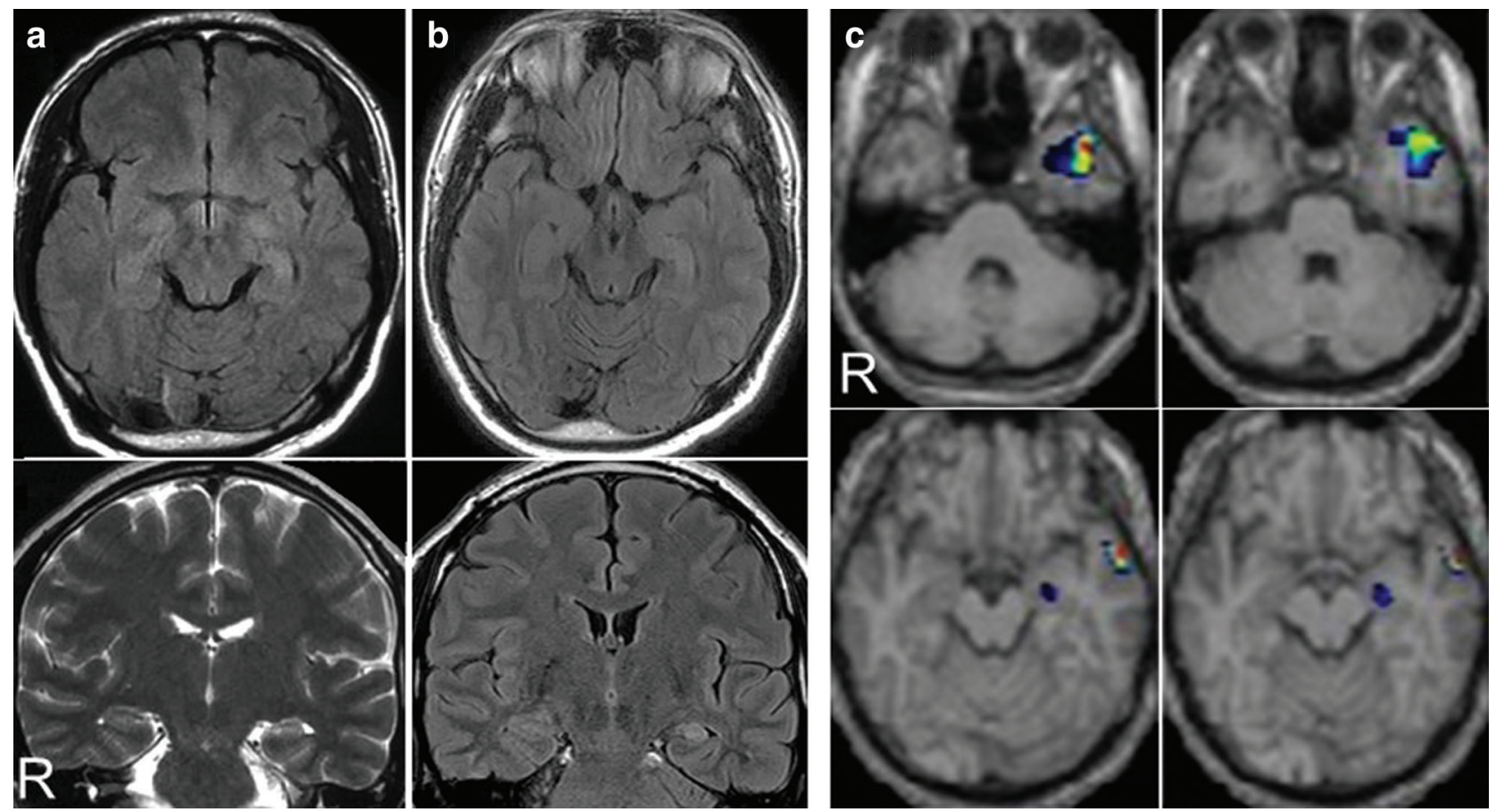

Fig. 2 MRI and interictal FDG-PET. Upper row is axial view and lower row is coronal view. At the onset of FIAS, MRI detected left temporal hippocampal atrophy in FLAIR image (upper row) and T2WI (lower row) (a). At the time of presurgical evaluation, left hippocampus showed atrophy with high intensity corresponding to HS in FLAIR image of MRI (b), and FDG-PET using statistical parametric mapping analysis revealed decreased glucose metabolization and perfusion in medial and anterior part of the left temporal lobe (c). FDG-PET: ${ }^{18} \mathrm{~F}$-fluorodeoxyglucose positron emission tomography, FIAS: focal impaired awareness seizure, FLAIR: fluid-attenuated inversion-recovery, HS: hippocampal sclerosis, MRI: magnetic resonance imaging, T2WI: T2-weighted image.

surgery, 12 years after NBD onset. Before and during evaluation, three more antiepileptic drugs (carbamazepine [500 mg/day], lacosamide [300 mg/day], and perampanel [10 $\mathrm{mg} /$ day]) were tried for 1 year, but they failed to control the seizures. Interictal
EEG showed multiple sharp waves in the left sphenoidal electrode and sometimes in the left anterior temporal lesion (F3) in long-term video-EEG study. There was no interictal epileptic discharge in the contralateral hemisphere. During a 5-day monitoring 

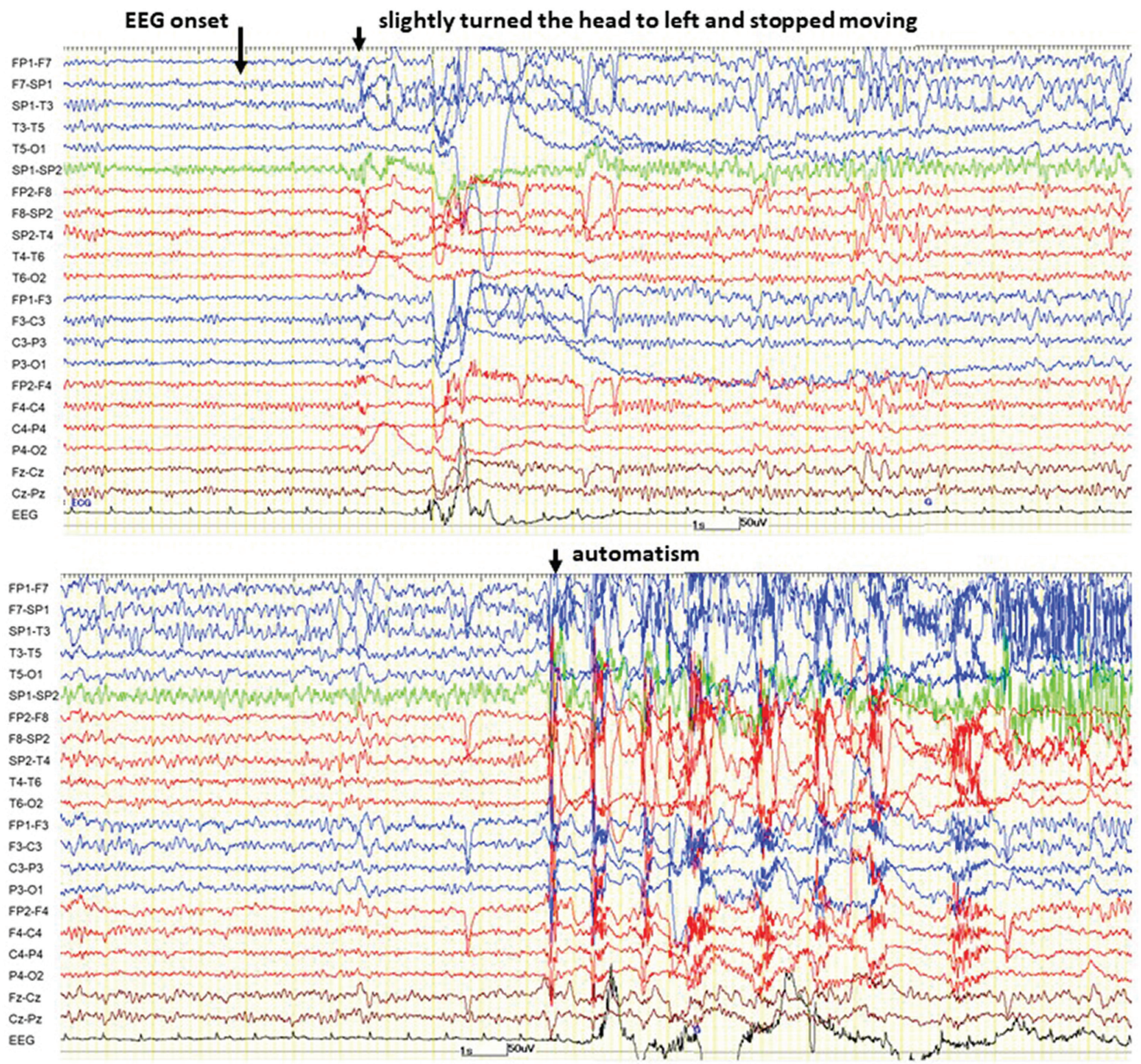

Fig. 3 Ictal scalp EEG on presurgical study. Epileptic discharge started on left SP1. EEG: electroencephalography, SP1: sphenoidal lead.

period under the off-drug condition, we could catch five ictal events. In the ictal state, the patient slightly turned his head to the left and stopped moving for some time while staring blankly to the left, followed by oroalimentary and gesture automatism. In one of these ictal events, the patient presented with automatism followed by rotation of the head to the right and tonic symptoms of the right upper and lower limbs, and progressed to FBTCS. All ictal EEGs showed a similar pattern of lateralized rhythmic sharp waves, which appeared in the left sphenoidal electrode followed by background activity change. This activity evolved and spread to the temporal tip and the contralateral medial temporal lobe (Fig. 3).
MRI in T2WI demonstrated shrinking and hyperintensity in the left hippocampus, and no findings were suggestive of NBD (Fig. 2b). ${ }^{18}$ F-fluorodeoxyglucose-position emission tomography (FDG-PET) showed low metabolism in the left medial and anterior temporal lobe (Fig. 2c). In the other area, FDG-PET did not reveal hypometabolism, which would have indicated hypofunction after NBD history. ${ }^{123}$ Iomazenil single photon emission computed tomography also showed similar results. Magnetoencephalography showed the scattering of equivalent dipole along the left Sylvian fissure. The Wechsler Adult Intelligence Scale-III (WAIS-III) found that the verbal intellectual quotient (VIQ) of the patient 

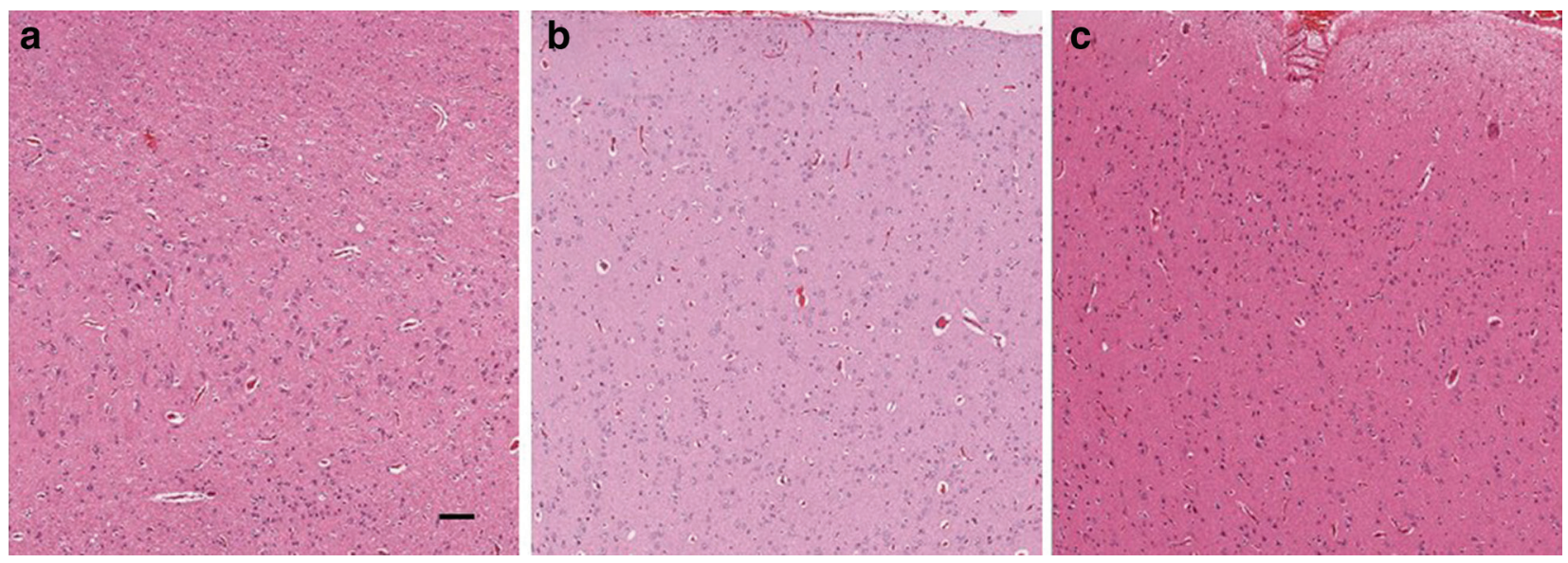

Fig. 4 Histopathological findings of hematoxylin-eosin staining on resected tissues. CA1 of left hippocampus showed cell loss in the pyramidal cell layer corresponding to HS (a). There were no specific inflammatory findings in hippocampus, parahippocampal gyrus (b) and inferior temporal gyrus (c). Horizontal bar indicates $100 \mu \mathrm{m}$. HS: hippocampal sclerosis.

was 58; performance IQ (PIQ) was 68, and full-scale IQ (FIQ) was 59. The Wechsler memory scale-revised (WMS-R) indicated that his verbal memory was 66; visual memory was 108; attention/concentration was 90, and delayed recall was 77 . The Wisconsin Card Sorting Test (WCST), used to evaluate frontal lobe function, showed that categories achieved (CA) was 3; perseverative errors of Nelson (PEN) was 9, and difficulty of maintaining set (DMS) was 1 . The patient's overall score was 45.4 in the quality of life in epilepsy inventory (QOLIE) -31-P. The patient was right-handed, and Wada test and functional MRI showed left-sided speech dominance. We diagnosed his seizure as left MTLE due to HS. While NBD was well controlled on low-dose steroid, the impact of the seizures on his social life was immense.

He had left ATL 13 years after NBD onset. Histopathological examination revealed left HS and the temporal cortex did not have findings related to NBD or any chronic inflammatory diseases (Fig. 4). Two to three months after surgery, the patient felt causeless frustration, and was temporarily managed with anxiolytics; his symptoms improved in a few months. Intelligence quotient improved, but his memory worsened after surgery. In neuropsychological tests 2 years after surgery, memory function in WMS-R has deteriorated considerably, such that verbal memory was 51; visual memory was 60; attention/concentration was 84, and delayed recall was 50. On the other hand, WAIS-III and WCST had risen considerably; 61, 90, and 78 in VIQ, PIQ, and FIQ, respectively; and 3, 9, and 0 in CA, PEN, and DMS, respectively. As for QOL in QOLIE-31-P, although cognitive function declined by 1.6 points, social function and total QOL increased by 12 points and 3.5 points, respectively, and the overall score increased to 62 . He had no major problems in his daily life, has been seizure-free for more than 2 years after surgery while being on two antiepileptic drugs (carbamazepine [500 mg/day] and lacosamide [300 mg/day]) and has been able to return to work with NBD well controlled.

\section{Discussion}

Seizures are rare in BD. The prevalence of seizure, excluding seizure occurrence during neurologic exacerbation, has been reported to be $2.2 \%$; the most common seizure type was FBTCS, and was accompanied by focal motor seizures. ${ }^{8)}$ The seizures were thought to be unrelated to neurological attacks in BD. ${ }^{9)}$ In our case, the bilateral mesial temporal lobe lesions were present during the first admission (first relapse) to our hospital, although the hippocampal finding at the time of initial NBD onset was unknown. At that time, it was difficult to determine whether the left hippocampal lesion was HS. Right mesial temporal lesion resolved after steroid and immunosuppressive therapy. Meanwhile, the left hippocampal atrophy stabilized for about 10 years. The HS finding was a common one, with no pathological findings suggestive of chronic inflammation in the hippocampus, and there have been no reports showing an association between NBD and HS. These results suggest that HS in this case was incidental to NBD.

In the reported case, certain considerations had to be made before surgery. First, epileptogenicity of NBD lesions was a possibility, especially since the lesions involved the right medial temporal lobe and left anterior temporal lobe. We found four 
Table 1 Reported seizure cases with hippocampal lesion in neuro-Behçet's disease

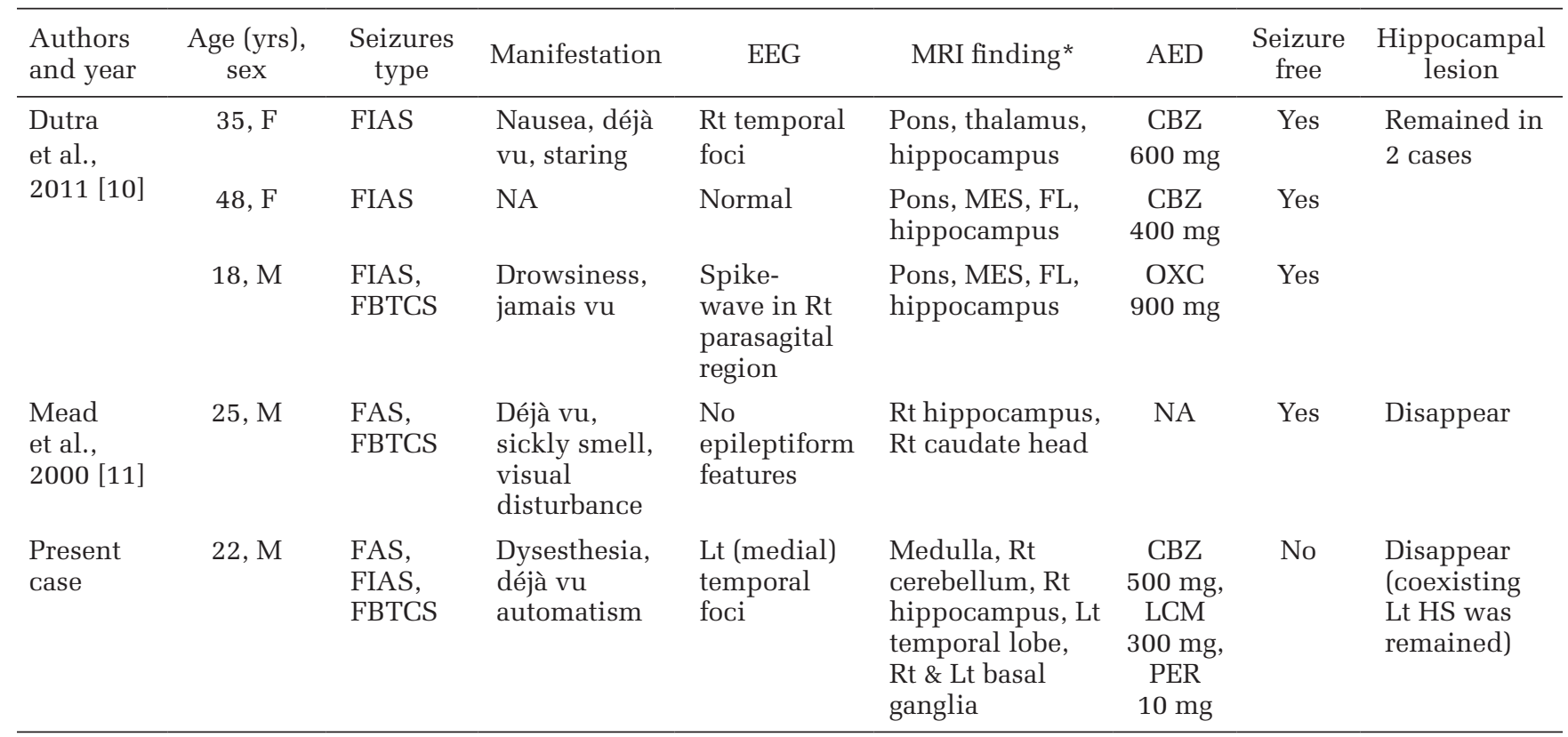

* Laterality of the lesions were inavairable in three cases. CBZ: carbamazepine, F: female, FAS: focal aware seizure, FBTCS: focal to bilateral tonic clonic seizure, FIAS: focal impaired awareness seizure, FL: frontal lobe, HS: hippocampal sclerosis, LCM: lacosamide, Lt: left, M: male, MES: mesencephalon, NA: not available, OXC: oxcarbazepine, PER: perampanel, Rt: right.

reported cases with hippocampal lesions in NBD (Table 1). ${ }^{10,11)}$ However, these patients who manifested focal seizures had good responses to antiepileptics, and one of the authors speculated that the seizures were possibly related to chronic inflammatory reaction to the small vessels that supply the mesial temporal lobe. ${ }^{10)}$ The lesions in the hippocampus reported in two of the four cases disappeared after steroid therapy, and no report of a resolved NBD lesion becoming epileptogenic was found. Second, elevation of IL-6 levels $>16.0 \mathrm{pg} / \mathrm{mL}$ has been proposed to be one of the diagnostic criteria of CP-NBD. ${ }^{5)}$ In our patient, the IL-6 in CSF was elevated (106 and $20.6 \mathrm{pg} / \mathrm{mL}$ ) during NBD relapse, indicating progressive disease. Our patient did not show the clinical symptoms and MRI findings corresponding to CP-NBD. In a previous study, IL-6 CSF levels were not significantly different between patients with acute NB and those with CP-NBD. ${ }^{3)}$ We determined that the patient was stable without showing signs of abnormally slow progression, although he had continued to take steroids to prevent symptom recurrence. Third, the risk of memory and cognitive deterioration if NBD recurred in the right mesial temporal lobe after ATL was considered. The symptoms of non-CP-NBD were, however, responsive to steroid therapy, with the attending neurologist confirming that the NBD symptoms were well controlled; and it was the epileptic seizures that most disturbed the patient's social life.

Surgery for MTLE with HS is an established treatment, and could lead to seizure remission in about $70-85 \%$ of patients with drug-resistant epilepsy. ${ }^{12)}$ Although the question of whether selective amygdalohippocampectomy (SAH) and ATL is more appropriate for MTLE, in terms of postoperative outcome and insult to cognitive function, has not been conclusively addressed. SAH is often selected on the side of the language-dominant hemisphere to reduce surgical impact on memory function. ${ }^{13)}$ Indication of SAH was also considered in our case. On the other hand, we were also concerned about the possibility that the anterior left temporal lobe was involved in the appearance of seizures because FIAS occurred after NBD lesions appeared in the anterior-medial left temporal lobe, and the generalized convulsions reoccurred with withdrawal of antiepileptic drugs. Hence, we planned an ATL in which the resection of the temporal lobe was reduced to the previous left temporal lobe lesion.

A multi-disciplinary team, including the patient, his family, and physicians discussed the aforementioned issues and everyone agreed to proceed with the surgery. Unfortunately, there was a strong decline in the patient's memory abilities, especially in visual memory. Visual memory is considered to be more 
prominent in disorders of the hippocampus in the right hemisphere, and the visual memory of this patient might have been mainly handled in the left temporal lobe. It is also possible that the right hippocampus became hypofunctional with a history of NBD lesions. Although hypometabolism was not evident on FDG-PET, it may be necessary to keep in mind that hypofunction may have occurred at the sites with a history of NBD lesions, as the majority of patients with NBD were reported to have some degree of neurocognitive decline. ${ }^{14)}$ While the patient has been free from seizures and NBD after the operation, with improvement in QOL, long-term follow-up is still necessary to evaluate the benefits of the surgical treatment. We could not find any previous case reports on surgical treatment of MTLE accompanied with NBD. The accumulation of similar cases will clarify the benefits and risks of surgical treatment in such patients.

\section{Conclusion}

We report the experience of a case of drug-resistant MTLE accompanied with NBD. Surgical treatment could be an option to control drug-resistant seizure symptoms in a patient with stable NBD, after weighing the benefits of the intervention and the potential risks.

\section{Conflicts of Interest Disclosure}

The authors have no conflicts of interest to declare.

\section{References}

1) Alpsoy E: Behçet's disease: a comprehensive review with a focus on epidemiology, etiology and clinical features, and management of mucocutaneous lesions. J Dermatol 43: 620-632, 2016

2) Kontogiannis V, Powell RJ: Behçet's disease. Postgrad Med J 76: 629-637, 2000

3) Ishido M, Horita N, Takeuchi M, et al.: Distinct clinical features between acute and chronic progressive parenchymal neuro-Behçet disease: meta-analysis. Sci Rep 7: 10196, 2017
4) Kalra S, Silman A, Akman-Demir G, et al.: Diagnosis and management of Neuro-Behçet's disease: international consensus recommendations. J Neurol 261: 1662-1676, 2014

5) Hirohata S, Kikuchi H, Sawada T, et al.: Clinical characteristics of neuro-Behcet's disease in Japan: a multicenter retrospective analysis. Mod Rheumatol 22: 405-413, 2012

6) Wiebe S, Blume WT, Girvin JP, Eliasziw M; Effectiveness and Efficiency of Surgery for Temporal Lobe Epilepsy Study Group: A randomized, controlled trial of surgery for temporal-lobe epilepsy. $N$ Engl J Med 345: 311-318, 2001

7) Yoon DL, Kim YJ, Koo BS, Kim YG, Lee CK, Yoo B: Neuro-behçet's disease in South Korea: clinical characteristics and treatment response. Int J Rheum Dis 17: 453-458, 2014

8) Aykutlu E, Baykan B, Serdaroglu P, Gökyigit A, Akman-Demir G: Epileptic seizures in Behçet disease. Epilepsia 43: 832-835, 2002

9) Kutlu G, Semercioglu S, Ucler S, Erdal A, Inan LE: Epileptic seizures in Neuro-Behcet disease: why some patients develop seizure and others not? Seizure 26: 32-35, 2015

10) Dutra LA, Braga-Neto P, Pedroso JL, et al.: Epilepsy and Behçet's disease: cortical and hippocampal involvement in Brazilian patients. J Neurol Sci 309: 1-4, 2011

11) Mead S, Kidd D, Good C, Plant G: Behçet's syndrome may present with partial seizures. J Neurol Neurosurg Psychiatry 68: 392-393, 2000

12) Lee SK: Treatment strategy for the patient with hippocampal sclerosis who failed to the first antiepileptic drug. J Epilepsy Res 4: 1-6, 2014

13) Wendling AS, Hirsch E, Wisniewski I, et al.: Selective amygdalohippocampectomy versus standard temporal lobectomy in patients with mesial temporal lobe epilepsy and unilateral hippocampal sclerosis. Epilepsy Res 104: 94-104, 2013

14) Fisher CA, Bernard C: A systematic review of neurocognitive functioning in Behçet's disease. Neuropsychol Rev 29: 498-521, 2019

Corresponding author: Ryosuke Hanaya, MD, PhD Department of Neurosurgery, Graduate School of Medical and Dental Sciences, Kagoshima University, 8-35-1 Sakuragaoka, Kagoshima, Kagoshima 890-8544, Japan.

e-mail: hanaya@m2.kufm.kagoshima-u.ac.jp 